\title{
Case Report \\ From Postural Orthostatic Tachycardia Syndrome to Radiologically Isolated Syndrome
}

\author{
Richa Tripathi ${ }^{1}$ and Evanthia Bernitsas $\mathbb{i D}^{2}$ \\ ${ }^{1}$ Emory University School of Medicine, Atlanta, GA, USA \\ ${ }^{2}$ Wayne State School of Medicine, Detroit, MI, USA \\ Correspondence should be addressed to Evanthia Bernitsas; ebernits@med.wayne.edu
}

Received 6 November 2017; Revised 15 January 2018; Accepted 17 January 2018; Published 25 February 2018

Academic Editor: Isabella Laura Simone

Copyright (c) 2018 Richa Tripathi and Evanthia Bernitsas. This is an open access article distributed under the Creative Commons Attribution License, which permits unrestricted use, distribution, and reproduction in any medium, provided the original work is properly cited.

\begin{abstract}
Background. Autonomic dysfunction is common in Multiple Sclerosis (MS) patients. Most spinal cord lesions entail some degree of autonomic nervous system dysfunction. MS patients may develop autonomic dysfunction later in their disease course. Methods. We report a patient with no prior history of MS presenting with orthostatic symptoms and diagnosed initially with postural orthostatic tachycardia syndrome (POTS). Four months later, she was diagnosed with radiologically isolated syndrome (RIS). The diagnosis was supported by imaging and CSF analysis. Conclusion. Our case sheds light on the need to consider autonomic dysfunction as an initial presentation of demyelinating pathology. Larger trials are needed to outline the possible association between POTS and RIS.
\end{abstract}

\section{Introduction}

Postural orthostatic tachycardia syndrome (POTS) is characterized by an orthostatic increase in the heart rate of $\geq 30$ beats per minute within 10 minutes of standing or during head-up tilt table test in the absence of orthostatic hypotension. Another standard for the pediatric group (12-19 years of age) is an increment of 40 beats per minute for 12-19 years of age group. The symptoms are due to cerebral hypoperfusion and autonomic overactivity and are relieved in recumbent position. Symptoms include orthostatic lightheadedness, tachycardia, fatigue, and mental clouding. POTS can be associated with central nervous system (CNS) lesions including demyelinating diseases [1-3]. It was thought that most Multiple Sclerosis (MS) cases with autonomic dysfunction are advanced in their clinical course; however, recent literature supports the presence of POTS in clinically isolated syndrome [4]. Management of the symptoms in such patients is usually delicate as most medications have several side effects.

\section{Case Presentation}

Our patient is a 30-year old woman, previously healthy, who presented to the outpatient cardiology clinic for episodes of postural lightheadedness and subjective tremulousness of her whole body. Head-up tilt table test was consistent with POTS. The remaining workup was normal. She was maintained on metoprolol $25 \mathrm{mg}$ twice a day and spironolactone $50 \mathrm{mg}$ daily. Four months later, she presented to the neurology clinic for recurrent episodes of lightheadedness, intermittent neck pain, and nausea.

Her neurological examination was unremarkable. She underwent Magnetic Resonance Imaging (MRI) scan of her cervical spine without contrast for her neck pain. It demonstrated T2 hyperintensities at C2 and C4-5 level, suspicious for demyelination (Figure 1). We further requested MRI scan of the brain with contrast, which showed several periventricular and pericallosal hyperintensities (Figure 2).

Cerebrospinal fluid (CSF) analysis showed 16 nucleated cells (97\% lymphocytes), high IgG index of 2.11 (normal limits 0-0.85), 21 CSF oligoclonal bands, and 1 serum oligoclonal band. Blood work including ANA, anti-dsDNA, ANNAT1, ANNAT2, and ANNAT3, PCCAB 1, 2, and 3, anti-Sm, anti-RNP, anti-SSA, anti-SSB, anti-Scl70, anti-Jol, ANCA, amphiphysin, striated muscle antibody, and CRMP-5 was normal. Serum NMO-IgG antibody was negative.

She was diagnosed with radiologically isolated syndrome (RIS) and she was started on glatiramer acetate. On follow-up, 


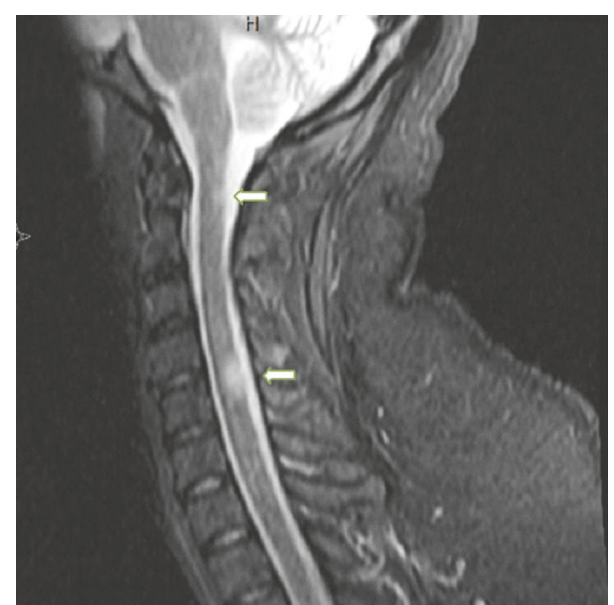

FIGURE 1: Sagittal T2-weighted images of the cervical spine showing abnormal signal cord at C2 and C4-5 level (arrows).

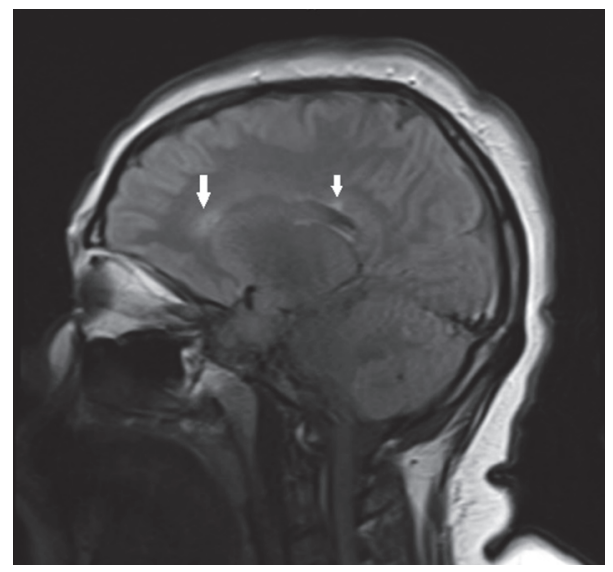

FIGURE 2: FLAIR sagittal images of the brain showing pericallosal hyperintensities. FLAIR: fluid- attenuated inversion recovery (arrows).

she reported occasional neck pain and continued to experience lightheadedness. One year later, she is clinically stable and she is being followed up in the MS clinic.

\section{Discussion}

An extensive literature review suggests that most patients develop POTS either several years prior to being diagnosed with MS or as a part of autonomic dysfunction associated with MS [1]. Our literature search revealed only one such case with pure autonomic dysfunction in the form of POTS as a presenting symptom of MS reported by Kuba et al. [5].

In a large retrospective study, Adamec et al. [6] reported a significant association between POTS and MS. Several mechanisms have been proposed for the development of these symptoms including sympathetic denervation and central causes such as sympathetic overdrive, hypovolemia, and involvement of the renin-angiotensin-aldosterone system. Sympathetic nervous system (SNS) dysfunction is a direct consequence of spinal cord injury and is due to loss of supraspinal control. It has been reported that the clinical symptomatology of SNS dysfunction is more common with higher spinal cord pathology above the level of mid thoracic cord. Furthermore, the higher the level, the greater the degree of severity of SNS dysfunction [7].

The Mayo Clinic experience study showed some correlation between autoimmunity and POTS. This study entails specific antibodies, such as ganglionic acetylcholine receptor antibody [8] and alpha-1 adrenergic receptor antibody that act as partial antagonists and induce reflex compensatory sympathetic activation [9].

Our patient was diagnosed with RIS that has not converted to clinically isolated syndrome (CIS) or definite MS yet. Her age and the presence of spinal cord lesions predict a higher risk for conversion to CIS or MS in the next 5 years [10]. In a recently published prospective, observational study, Habek et al. [4] reported that POTS was present in $9.5 \%$ of patients with clinically isolated syndrome (CIS). In this study, age and POTS were found to predict early conversion from CIS to MS.

Up till now, there are no case reports or studies focused on a possible association between RIS and POTS, and the role of POTS in predicting conversion from RIS to CIS or definite MS is still unknown. Our case report highlights the need for further studies to explore the presence and significance of POTS in RIS.

\section{Conflicts of Interest}

The authors declare that they have no conflicts of interest.

\section{References}

[1] K. Kanjwal, B. Karabin, Y. Kanjwal, and B. P. Grubb, "Autonomic dysfunction presenting as postural orthostatic tachycardia syndrome in patients with multiple sclerosis," International Journal of Medical Sciences, vol. 7, no. 2, pp. 62-67, 2010.

[2] R. Satish Raj, "The postural tachycardia syndrome (POTS): pathophysiology, diagnosis management," Indian Pacing Electrophysiol J, vol. 6, no. 2, pp. 84-99, 2006.

[3] R. Freeman, W. Wieling, F. B. Axelrod et al., "Consensus statement on the definition of orthostatic hypotension, neurally mediated syncope and the postural tachycardia syndrome," Autonomic Neuroscience: Basic \& Clinical, vol. 161, no. 1-2, pp. 46-48, 2011.

[4] M. Habek, M. Krbot Skorić, L. Crnošija, T. Gabelić, B. Barun, and I. Adamec, "Postural orthostatic tachycardia predicts early conversion to multiple sclerosis after clinically isolated syndrome," European Neurology, vol. 77, no. 5-6, pp. 253-257, 2017.

[5] H. Kuba, K. Arakawa, T. Taniwaki, Y. Maeda, T. Yamada, and J.-I. Kira, "A case of relapsing multiple sclerosis presenting with only autonomic symptoms including orthostatic hypotension, hiccups and vomiting," Journal of Clinical Neurology, vol. 39, no. 9, pp. 930-934, 1999.

[6] I. Adamec, M. Lovrić, D. Žaper et al., "Postural orthostatic tachycardia syndrome associated with multiple sclerosis," Autonomic Neuroscience: Basic and Clinical, vol. 173, no. 1-2, pp. 6568, 2013. 
[7] R. W. Teasell, J. M. O. Arnold, A. Krassioukov, and G. A. Delaney, "Cardiovascular consequences of loss of supraspinal control of the sympathetic nervous system after spinal cord injury," Archives of Physical Medicine and Rehabilitation, vol. 81, no. 4, pp. 506-516, 2000.

[8] M. J. Thieben, P. Sandroni, D. M. Sletten et al., "Postural orthostatic tachycardia syndrome: The Mayo Clinic experience," Mayo Clinic Proceedings, vol. 82, no. 3, pp. 308-313, 2007.

[9] H. Li, X. Yu, C. Liles et al., "Autoimmune basis for postural tachycardia syndrome., Journal of the American Heart Association, vol. 3, no. 1, p. e000755, 2014.

[10] D. T. Okuda, A. Siva, O. Kantarci et al., "Radiologically isolated syndrome: 5-year risk for an initial clinical event," PLoS ONE, vol. 9, no. 3, Article ID e90509, 2014. 


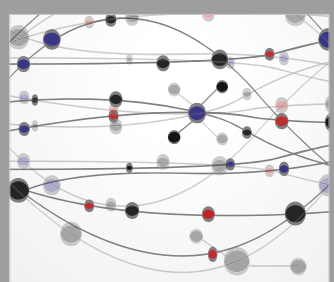

The Scientific World Journal
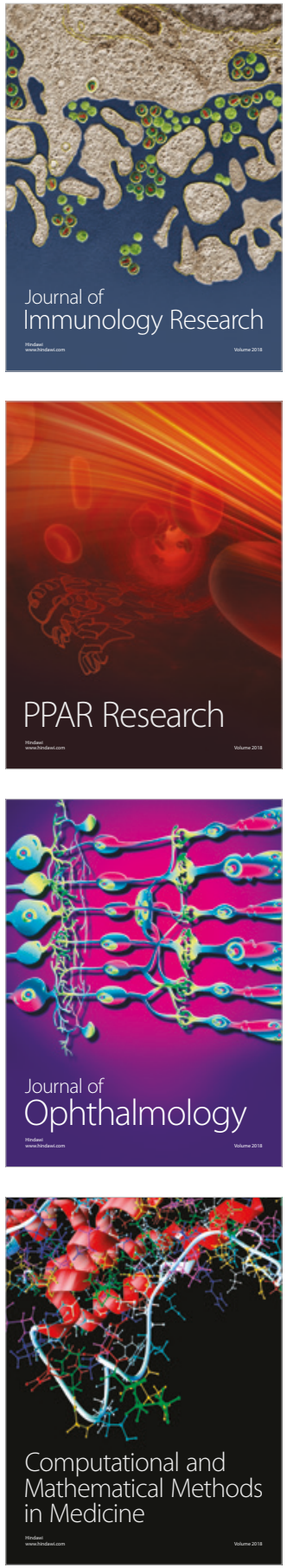

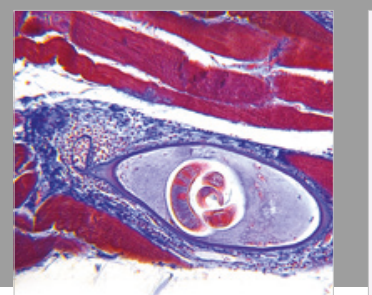

Gastroenterology Research and Practice

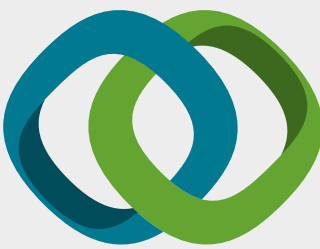

\section{Hindawi}

Submit your manuscripts at

www.hindawi.com
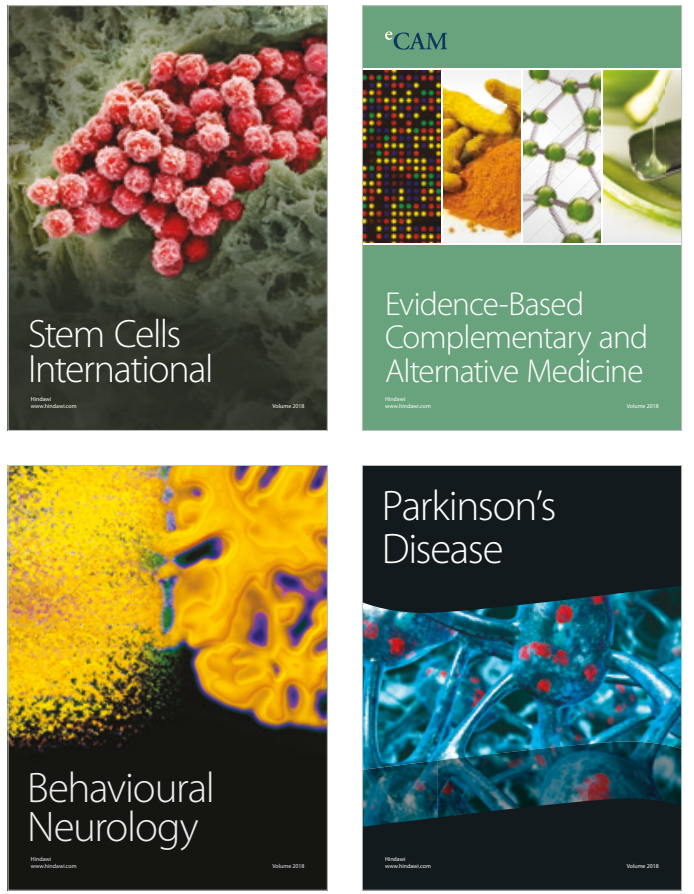

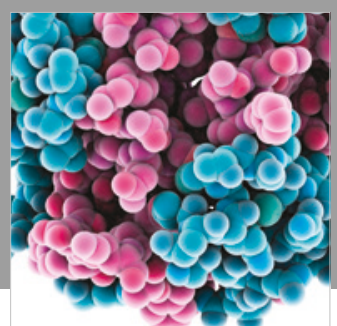

ournal of

Diabetes Research

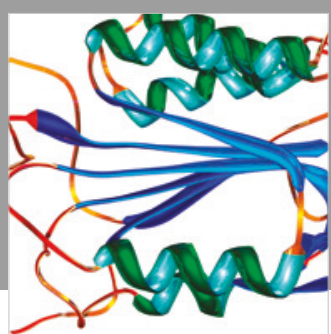

Disease Markers
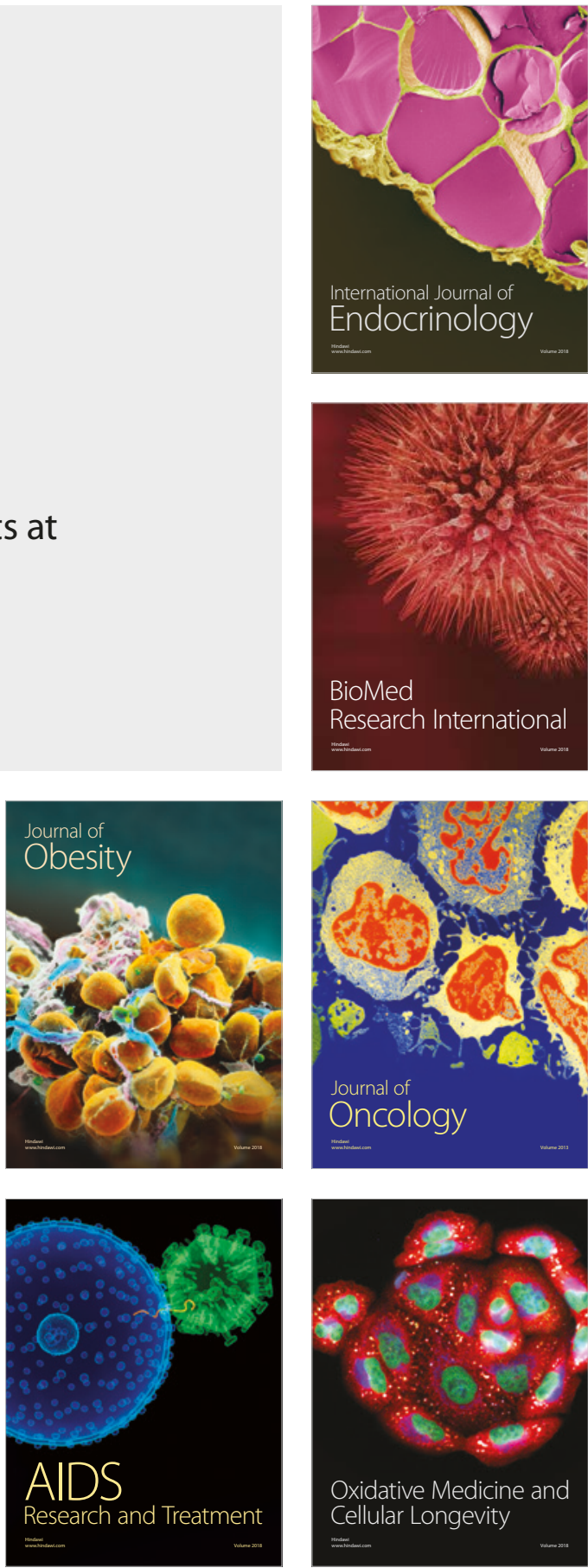